\title{
Os motivos de procura pelo Pronto Socorro Pediátrico de um Hospital Universitário referidos pelos pais ou responsáveis
}

\author{
The reasons to look for a university hospital's childish medical \\ treatment which is requested by parents or somebody responsible \\ for the child
}

\author{
Simoni Batistela ${ }^{1}$; Natália Paludeto Guerreiro²; Edilaine Giovanini Rossetto ${ }^{3}$
}

Resumo

Os serviços de saúde são organizados, hierarquicamente, em três níveis de complexidade tecnológica: primário, secundário e terciário, e a população deveriam acessá-los conforme a especificidade de suas queixas, visando, dessa forma, maior eficiência na utilização dos recursos e universalização do acesso. Na prática, percebe-se que a população prefere recorrer a hospitais de nível terciário para situações corriqueiras, as quais poderiam ser solucionadas nas Unidas Básicas de Saúde (UBSs). De acordo com o problema acima descrito, esse levantamento teve como objetivo avaliar os motivos que levaram os pais ou responsáveis a procurarem um hospital de alta complexidade para atendimento das crianças, investigando a freqüência e finalidade que essa população utiliza os serviços básicos de saúde. A presente pesquisa foi realizada com os pais ou responsáveis pelas crianças atendidas em um Pronto Socorro Pediátrico de um Hospital Universitário, localizado na cidade de Londrina, sendo aplicado entrevista a 222 usuários durante os meses de março a abril de 2006. Após análise dos dados, observouse que, apesar de $80,6 \%$ da população estudada referirem freqüentar as UBSs, $56,7 \%$ dos atendimentos no Pronto Socorro Pediátrico ocorreram mediante procura direta e 33,3\% dos entrevistados elegeram a qualidade no atendimento como motivo para justificar essa procura. A queixa mais freqüente foi à febre com $21,6 \%$ e as causas externas apareceram em último lugar com apenas $1,4 \%$. Por meio desse estudo, pôde-se concluir que o Pronto Socorro Pediátrico do Hospital Universitário de Londrina recebe grande contingente de crianças com queixas, muitas vezes, passíveis de resolverem-se em nível básico de atendimento à saúde, porém, os hospitais, de um modo geral, possuem maior legitimidade do que as UBSs diante da população habituada ao longo dos anos a recorrer aos serviços hospitalares.

Palavras-chave: Saúde da criança. Atenção básica. Pronto socorro infantil. Acesso aos serviços de saúde.

\begin{abstract}
Health services are managed hierarchically in three levels of technological complexity: primary, secondary and tertiary, and the population should use them according to the specificity of their complaints, aiming at more efficiency and universal access. In practice, the population prefers to go to tertiary level hospitals for simple problems, which could easily be solved by Basic Health Service Units (BHUs). The objective of this survey was to find out the reasons why parents or children caretakers look for high complexity hospitals, and how frequently they make use of basic health services. This
\end{abstract}

1 Graduanda do $4^{\circ}$ ano do curso de enfermagem da Universidade Estadual de Londrina; Bolsista IC/UEL durante o ano de 2005. Endereço: Rua 30, 2660, Barretos - SP. Fone: (17)3322 8272. E-mail: simoni_batistela@yahoo.com.br.

2 Graduanda do $4^{\circ}$ ano do curso de enfermagem da Universidade Estadual de Londrina.

3 Mestre em enfermagem fundamental pela Universidade de São Paulo (USP); docente do departamento de enfermagem da Universidade Estadual de Londrina. 
research interviewed 222 parents and caretakers of children who were undergoing medical treatment at the Pediatrics Emergency Room from a School Hospital located in the city of Londrina, in March and April of 2006. Data analysis showed that, although $80,6 \%$ of the population interviewed used the BHUs , 56,7\% of the children seen at the Pediatrics Emergency Room came with no referrals, and 33,3\% chose the ER for its quality services. The most frequent complaint was fever $(21,6 \%)$ and other external causes took the last position with only $1,4 \%$ of the cases . Results from the study showed that the Londrina State University School Hospital Pediatrics Emergency Room sees a great number of children with simple complaints which could be easily solved by basic level health care. However, in general, most hospitals have been legitimized by the population, used to always look for hospitals, rather than BHUs, whenever they need any kind of health care.

Keywords: Children health. Basic health care. Pediatrics Emergency Room. Medical treatment.

\section{Introdução}

Os serviços de saúde devem ser organizados de forma a garantir amplo acesso e total cobertura à população em seus três níveis de atenção: primário, secundário e terciário, de acordo com o tipo de atendimento e a complexidade do serviço. Com a implantação plena do Sistema Único de Saúde (SUS), o modelo de assistência à saúde seria como uma pirâmide: na base, estariam localizadas as unidades básicas de saúde responsáveis pela atenção primária, na parte intermediária encontrarse-iam os serviços ambulatoriais que atendem em nível secundário e, no topo, estariam os serviços hospitalares de maior complexidade atendendo o nível terciário (CECILIO, 1997).

As unidades básicas de saúde deveriam funcionar como portas de entrada ao sistema, oferecendo grande resolutividade e assistência integral, cabendo aos hospitais e ambulatórios atendimentos que exijam especialidades ou maior densidade tecnológica. No entanto, na prática, isso não ocorre e a porta de entrada tem sido os hospitais, seja através dos prontos socorros ou ambulatórios. Cecílio (1997) relata que os atendimentos hospitalares são expressivamente maiores do que o atendimento feito nas unidades básicas de saúde, na maioria dos municípios onde há acesso hospitalar. De acordo com Travassos e Martins (2004) os fatores determinantes do acesso aos serviços de saúde estão, geralmente, relacionados à necessidade de saúde, aos usuários, aos prestadores de serviços, à organização e à política local.
Segundo a Agenda de compromissos para saúde integral da criança e redução da mortalidade infantil, a organização de uma rede integrada de assistência deve ser baseada nos princípios já assegurados pela legislação (Constituição, Estatuto da Criança, e Sistema Único de Saúde) garantindo o acesso aos serviços de saúde hierarquizados, enfocando a integralidade do indivíduo e da assistência. Este documento aponta alguns princípios norteadores do cuidado à saúde da criança, como o acesso universal, acolhimento, responsabilização, assistência integral e resolutiva, equidade entre outros. Se as unidades básicas se comprometessem com esses princípios, talvez houvesse possibilidade de mudanças no cenário de busca aos serviços de saúde e a população procuraria as UBSs como porta de entrada para a rede de saúde, deixando os hospitais e ambulatórios para os atendimentos mais complexos (BRASIL, 2005).

Há uma crença por parte dos pais de que, nos hospitais, os filhos estariam mais bem amparados que na rede básica, por isso, preferem ir até os hospitais por problemas que seriam resolvidos em nível básico, além deste fato, há que se considerar o tempo de espera para o atendimento pelo pediatra na UBS e a falta de hábito da criança ser consultada pela enfermeira. A demanda espontânea para os hospitais justificaria-se apenas nos horários em que as unidades básicas não estariam funcionando e para os casos de urgência e emergência (KOVACS et al., 2005). 
Desde a Segunda Guerra Mundial, o hospital foi considerado a peça central do sistema de saúde e a assistência embasada por um modelo médico que privilegiava a medicina curativa. Esse modelo assistencial hospitalocêntrico com utilização irracional dos recursos tecnológicos, pouca resolutividade e integralidade prevalece até os dias de hoje. Nesse contexto, a rede básica é vista como desqualificada e descartável, passando a porta de entrada para níveis de maior densidade tecnológica (ROCHA et al., 1998).

Diante do exposto acima, foram objetivos deste estudo avaliar juntos aos pais ou responsáveis o motivo pela escolha de um hospital de alta complexidade para o atendimento aos filhos, investigando, ao mesmo tempo, a freqüência que a população utiliza os serviços básicos de saúde e com que finalidade o faz.

\section{Material e método}

Sob uma abordagem quantitativa, desenvolveuse esse estudo no Pronto Socorro Pediátrico (PSP) do Hospital Universitário Regional Norte do Paraná (HURNP) localizado na cidade de Londrina.

A população estudada foi composta por crianças com idade entre 0 e 11 anos e 11 meses que procuraram o Pronto Socorro Pediátrico durante o período de 06 de março a 07 de abril de 2006. De acordo com Cruz (2005), no ano de 2004, 8848 crianças passaram pelo Pronto Socorro Pediátrico, e, com base nesse número, foi calculado uma média mensal de atendimentos dividindo-se o total (8848) por 12 (meses). Foi definida uma amostra representativa da população como $30 \%$ do total de atendimentos num mês, resultando em 222 entrevistas.

A pesquisa foi iniciada após ser submetida e aprovada pelo Comitê de Bioética da Universidade Estadual de Londrina.

A coleta de dados foi realizada por meio de questionários aplicados pelas pesquisadoras aos pais ou responsáveis pelas crianças que procuraram o Pronto Socorro Pediátrico. Após explicação da pesquisa, os participantes que concordaram em participar assinaram um termo de consentimento livre e esclarecido.

Antes da coleta propriamente dita, foi realizado um teste piloto durante uma semana para avaliação da viabilidade do questionário, quando foram realizados os ajustes necessários.

Os dados coletados foram transcritos para um banco de dados elaborado no programa Excel e, posteriormente, foram tabulados para análise e avaliação de freqüência não sendo realizado nenhum tipo de teste estatístico. Os resultados foram apresentados por meio de tabelas.

\section{Apresentação dos resultados}

Entre as 222 crianças participantes desta pesquisa, 94,14\% $(n=209)$ foram trazidas ao Pronto Socorro Pediátrico do Hospital Universitário (PSP/ $\mathrm{HU})$ pelos pais, sendo as demais acompanhadas por avós, tios, entre outros.

Quando questionado se a procura pelo PSP/HU foi direta ou os acompanhantes passaram anteriormente por outros serviços de saúde, $56,76 \%(\mathrm{n}=126)$ das crianças vieram direto ao PSP/HU e 43,24\% $(n=96)$ foram levadas a outros serviços de saúde antes de serem trazidas ao PSP/HU. Dentre as 96 crianças que estiveram anteriormente em outros serviços, $60,54 \%$ receberam algum tipo de encaminhamento para hospital universitário.

Na Tabela 1, observam-se somente os 43,24\% $(n=96)$ da população que procuraram por outros serviços antes de procurarem o PSP/HU. O Pronto Atendimento Infantil (PAI), serviço municipal, de nível secundário, que atende crianças 24 horas por dia foi o local mais procurado antes do HU (29,17\%). Em segundo lugar, 22,92\% dos acompanhantes referiram as Unidades Básicas de Saúde (UBS) como serviço utilizado antes do PSP/HU, seguido de 20,83\% em outros serviços como hospitais de nível 
secundário, Ambulatórios do Hospital das Clínicas (AHC), dentre outros. As crianças provenientes de outras cidades próximas ao município de Londrina ocuparam 16,66\%. Encontrou-se ainda 10,42\% dos acompanhantes que peregrinaram com suas crianças doentes por pelo menos três serviços de saúde até terem seus filhos atendidos no PSP/HU.

Tabela 1. Distribuição dos serviços de saúde procurados pelos pais ou responsáveis das crianças antes do atendimento do Pronto Socorro Pediátrico de um Hospital Universitário. Londrina, 2006.

\begin{tabular}{lcc}
\hline \multicolumn{1}{c}{ Serviços de saúde procurados pelas crianças antes do PSP/HU } & $\mathbf{N}$ & $\mathbf{\%}$ \\
\hline Pronto Atendimento Infantil & 28 & 29,17 \\
Unidades Básicas de Saúde & 22 & 22,92 \\
Outros serviços & 20 & 20,83 \\
Cidades próximas à Londrina & 16 & 16,66 \\
Mais de 2 serviços & 10 & 10,42 \\
\hline Total & 96 & 100,00 \\
\hline
\end{tabular}

Na Tabela 2, entre os 126 pais ou responsáveis que responderam ter vindo direto ao $\mathrm{PSP} / \mathrm{HU}$, $33,34 \%$ alegaram qualidade do atendimento como motivo para eleger esse serviço como primeira opção, seguido de $30,15 \%$ que escolheram o PSP/ HU devido às crianças fazerem tratamento ou acompanhamento prévionos ambulatórios do próprio HU ou do Hospital das Clínicas (AHC), órgão de nível secundário também vinculado a Universidade Estadual de Londrina (UEL). Já 17,47\% optaram pelo PSP/HU devido à acessibilidade geográfica, outros 9,52\% argumentaram diferentes motivos pela preferência, entre eles, familiares ou a própria pessoa que trabalha no HU o que facilitaria o atendimento, horário de funcionamento das UBSs, também sendo incluídas neste grupo as crianças trazidas pelos serviços de urgência (SIATE ou SAMU) que foram apenas três. A não resolutividade de outros serviços foi apontada por $9,52 \%$ dos entrevistados.

Tabela 2. Distribuição dos motivos referidos pelos pais ou responsáveis para procura direta do Pronto Socorro Pediátrico de um Hospital Universitário. Londrina, 2006.

\begin{tabular}{lcc}
\hline \multicolumn{1}{c}{ Motivo referido pela procura direta ao PSP/HU } & N & \% \\
\hline Qualidade do atendimento & 42 & 33,34 \\
Tratamento ou acompanhamento prévio & 38 & 30,15 \\
Acessibilidade geográfica & 22 & 17,47 \\
Não resolutividade de outros serviços & 12 & 9,52 \\
Outros & 12 & 9,52 \\
\hline Total & 126 & 100,00 \\
\hline
\end{tabular}

A Tabela 3 apresenta as queixas das crianças que levaram os acompanhantes a procurarem um serviço de saúde. Nesta questão, foram consideradas respostas que referiram mais de uma queixa pela mesma criança, por isso, o total ser um n=342 e não 222. Do total estudado, a febre foi o sintoma mais freqüente $(21,64 \%)$, seguido de $18,13 \%$ com queixas de vômitos ou criança que não conseguiam beber ou mamar e 15,20\% com dor. Alguns entrevistados (6,14\%) foram ao $\mathrm{PSP} / \mathrm{HU}$ por doenças previamente diagnosticadas, e, neste item, enquadram-se crianças portadoras 
de doenças crônicas (cardiopatia, renal crônico, Diabetes Mellitus descompensado, encefalopatias) e crianças que fazem acompanhamento ambulatorial (como doenças hematológicas, hérnia, atresia de esôfago, refluxo gastroesofágico ou tumores). Outros problemas de saúde referidos foram muito diversificados e, portanto, pouco freqüentes, como por exemplo: alergias (respiratórias, urticárias, dermatites), infecções ou inflamações (otite, conjuntivite, tendão inflamado), manchas no corpo, cistos dentre outros, portanto, foram agrupados num item denominado de outros, com uma incidência de $8,18 \%$.

Foi perguntado aos participantes qual o serviço de saúde de primeira escolha quando a criança adoece e $42,34 \%$ indicaram a UBS, seguido do PSP/ HU com $36,04 \%$, PAI com $13,96 \%$ e $7,66 \%$ dos entrevistados apontaram outros serviços, tais como, outros hospitais da cidade e os da região conforme se observa na Tabela 4.

Tabela 3. Distribuição das queixas apresentadas pelas crianças segundo o relato dos pais ou responsáveis no momento da procura pelo Pronto Socorro Pediátrico de um Hospital Universitário. Londrina, 2006.

\begin{tabular}{lcc}
\hline \multicolumn{1}{c}{ Queixas referidas pelos pais/responsáveis das crianças } & $\mathbf{n}$ & $\mathbf{\%}$ \\
\hline Febre & 74 & 21,64 \\
Vomita tudo que ingere ou não consegue beber ou mamar & 62 & 18,13 \\
Dor & 52 & 15,20 \\
Respiração rápida ou tosse & 32 & 9,36 \\
Doenças previamente diagnosticadas ou doenças crônicas & 21 & 6,14 \\
Diarréia & 20 & 5,85 \\
Letargia & 14 & 4,10 \\
Convulsão & 13 & 3,80 \\
Retorno agendado & 11 & 3,22 \\
Icterícia & 10 & 2,92 \\
Causas externas (traumas) & 5 & 1,46 \\
Outros & 28 & 8,18 \\
\hline Total & 342 & 100,00 \\
\hline
\end{tabular}

Tabela 4. Distribuição dos serviços de saúde de primeira escolha quando a criança adoece referida pelos pais ou responsáveis das crianças atendidas no Pronto Socorro Pediátrico de um Hospital Universitário. Londrina, 2006.

\begin{tabular}{lcc}
\hline Serviços de primeira escolha quando a criança adoece & N & \% \\
\hline UBS- & 94 & 42,34 \\
HU & 80 & 36,04 \\
PAI & 31 & 13,96 \\
Outros & 17 & 7,66 \\
\hline Total & 222 & 100,00 \\
\hline
\end{tabular}


Em relação à atenção básica, foi questionado se a população estudada utilizava-se deste recurso, e 19,37\% ( $\mathrm{n}=43)$ dos 222 entrevistados assumiram que não utilizavam, contra 80,63\% $\quad(n=179)$ que confirmaram serem usuários das UBS para atendimento de suas crianças. Dentre os $80,63 \%$ que responderam afirmativamente, perguntou-se para qual finalidade a criança é levada à unidade. A maioria dos entrevistados apresentou mais de uma justificativa, o que explica um total de 253 ao invés de 179 , sendo que a procura por pediatra foi responsável pela metade das respostas com 49,80\% e esta forma de acesso é, geralmente, por meio de consultas previamente agendadas e com finalidade de acompanhar o crescimento e desenvolvimento infantil. Em segundo lugar, apareceram outras ações consideradas como básicas com 36,37\%. Por ações básicas, entende-se vacinação (campanhas ou não) e puericultura, que avalia o crescimento e desenvolvimento, acompanha e orienta a alimentação da criança e atualiza e completa o quadro vacinal, além de inalação. A procura por outros profissionais como fisioterapeutas e dentistas ocupou o último lugar com 13,83\% das respostas, sendo que a fisioterapia atende somente por encaminhamento de outros profissionais de saúde e a procura pela odontologia geralmente é espontânea (Tabela 5).

Tabela 5. Distribuição dos motivos de procura pelas Unidades Básicas de Saúde referidos pelos pais ou responsáveis das crianças atendidas no Pronto Socorro Pediátrico de um Hospital Universitário. Londrina, 2006.

\begin{tabular}{lcc}
\hline \multicolumn{1}{c}{ Serviços utilizados pelas crianças nas UBS } & N & \% \\
\hline Pediatra & 126 & 49,80 \\
Ações básicas & 92 & 36,37 \\
Outros & 35 & 13,83 \\
\hline Total & 253 & 100,00 \\
\hline
\end{tabular}

\section{Discussão}

Esse estudo investigou os motivos pelos quais os pais ou responsáveis pelas crianças atendidas no Pronto Socorro Pediátrico do Hospital Universitário do município de Londrina escolheram tal instituição para prestação de serviço em saúde. Dentre os 222 entrevistados, 94\% das crianças foram trazidas pelos pais, na maioria das vezes, as mães que estiveram como acompanhantes (MARTIN; ANGELO, 1999).

Com relação à procura por um serviço de urgência, mais da metade das crianças $(56,7 \%)$ foram trazidas diretamente ao PSP/HU, ou seja, sem serem referidas por outros serviços. Quando a população utiliza um pronto socorro como porta de entrada em um sistema de saúde, obtém um atendimento geralmente restrito a queixa e sem enfoque para integralidade da assistência, além do que, a grande procura por um serviço de urgência sem encaminhamentos pode prejudicar o atendimento dos casos considerados adequados ou de urgência já que o aumento da demanda gera acúmulo de tarefas, sobrecarga profissional e aumento dos gastos hospitalares (FURTADO; ARAÚJO; CAVALCANTI, 2004).

Os 43,3\% restantes da população, tiveram que procurar por pelo menos dois serviços em busca da solução de suas enfermidades. Destaca-se ainda que 10 entrevistados referiram ter sido necessário procurar o PSP/HU após passar por dois outros serviços de saúde. Tais resultados sugerem uma baixa resolutividade e eficácia dos serviços que atendem a criança. Malta et al. (2004) colaboram com essa discussão afirmando que quando há fragmentação do cuidado é o usuário quem faz seu próprio caminho pelas redes de serviço, ocasionando uma prática inadequada podendo levar a erros diagnósticos, acesso negado e não efetividade do atendimento. 
Vale ressaltar que dentre os 96 pacientes vindos de outros serviços de saúde, 63,5\% apresentaram algum tipo de encaminhamento (por telefone ou escrito), sendo que essa seria a forma ideal de se chegar a um hospital de nível terciário, após tentar um serviço de menor nível de complexidade tecnológica.

Dos 96 usuários que procuraram outros serviços antes do atendimento no PSP/HU, 29,1 \% foram ao Pronto Atendimento Infantis serviços municipais de nível secundário de complexidade tecnológica com atendimento 24 horas e apenas 22,9\% (dos 96 entrevistados) passaram pelo nível primário de atenção antes de chegarem ao pronto socorro pediátrico. $\mathrm{O}$ esperado seria encontrar um número maior de crianças utilizando-se da rede básica como porta de entrada para o sistema de saúde. Porém, Cecilo (1997) defende em seu estudo que a população habituou-se a buscar o atendimento básico apenas em situações específicas, por meio de atendimento agendado, não o fazendo para outras ocasiões.

No levantamento dos motivos pelos quais os acompanhantes preferiram levar as crianças diretamente ao $\mathrm{PSP} / \mathrm{HU}, 33,3 \%$ apontaram bom atendimento do serviço. Segundo os entrevistados, esse fato é justificado por ser um hospital-escola, com presença de alunos e professores, além de outros profissionais como médicos e enfermeiros, o que contribui para uma boa assistência. Além disso, segundo os participantes da pesquisa, o hospital tem tecnologia avançada que possibilita realização de exames trazendo, portanto, resolutividade para os problemas. Entre as respostas que motivaram a escolha do PSP/HU como primeira opção, também surgiu proximidade do HU à residência da população com 17,4\%. A existência de um hospital de alta complexidade nas imediações motiva a população residente na área a levar as crianças ao hospital, já que este possui mais recursos humanos, físicos, material e diagnóstico. Nesta questão, ficou claro o total desconhecimento por parte da população sobre a função dos diferentes níveis de complexidade dos serviços de saúde e sobre a organização dos mesmos, gerando mais desorganização. Dados semelhantes foram encontrados em estudo realizado em Recife por Kovacs et al. (2005), no qual os motivos escolhidos pela procura direta por pronto socorro foram: qualidade geral do atendimento, acessibilidade geográfica e qualidade da atenção médica.

No presente trabalho, 30,15\% das 126 crianças levadas diretamente ao PSP faziam acompanhamento ou tratamento ambulatorial na instituição. Esse grupo referia-se àquelas crianças que apresentavam alguma queixa diferente dos sinais da patologia em acompanhamento ou tratamento ou aquelas cujos médicos responsáveis marcavam retorno ambulatorial no PSP. Na primeira particularidade, os acompanhantes justificaram a escolha pelo fato das crianças já serem acompanhadas pelo serviço e conhecidas pelos profissionais. $\mathrm{Na}$ segunda ocorrência, observa-se uma alteração do papel de um pronto socorro que, ao invés de ser destinado a atender situações de urgência, é utilizado como um ambulatório.

Nesse estudo, verificou-se que grande parte das queixas apresentadas pelos pais ou responsáveis correspondia aos sinais e sintomas abordados pelo protocolo de Atenção Integrados as Doenças Prevalentes da Infância (AIDPI). A febre foi o sintoma que mais freqüentemente motivou os pais a procurar por um hospital (21,6\%). Baracat (2005) confirma os dados encontrados no PSP de Londrina quando refere que as queixas febris correspondem até $20 \%$ do total de atendimentos em serviços de emergências. Ainda de acordo com o referenciado autor, a febre é o sintoma mais frequente na criança doente, porém, não é um sinal específico e apenas se o quadro febril persistir por mais de sete dias fazse necessário o encaminhamento para serviços de maior complexidade para investigação.

A segunda queixa mais freqüente foi vomita tudo que ingere ou não consegue beber ou mamar com 
18,1\% das respostas. Respiração rápida ou tosse, diarréia, letargia e convulsão também estiveram presentes em freqüências menores. Dentre os 222 responsáveis entrevistados, $15,2 \%$ referiram a dor como motivo pela procura por atendimento no PSP/ HU.

A tabela 3 evidencia também que $6,1 \%$ das queixas foram decorrentes das doenças previamente diagnosticadas ou doenças crônicas. Esses casos requerem tratamento prolongado e, na maioria das vezes, há necessidade de reinternações freqüentes seja para exames, tratamento paliativo ou algum episódio de crise. O hospital assume papel importante no decorrer deste processo e os responsáveis por estas crianças elegem o pronto socorro como referência, pelo fato de fazerem acompanhamentos nos ambulatórios da instituição e, muitas vezes, a rede básica desconhece a presença ou evolução dos casos (VIEIRA; LIMA, 2002).

As causas externas concentraram uma proporção muito pequena da procura pelo pronto socorro. Das 222 crianças atendidas, apenas 5 procuraram em decorrência de acidentes ou traumas, enquanto um pronto socorro deveria receber majoritariamente casos de urgência e emergência. As 5 ocorrências dividiram-se em: 2 quedas, 2 atropelamentos e 1 ingestão excessiva de medicamento. Filácomo et al. (2002) encontraram resultados semelhantes, sendo que a incidência de acidentes da infância ocupou $9,72 \%$ dos atendimentos e a principal causa foi quedas, assim como o encontrado no PSP/HU.

Foi abordado junto aos acompanhantes qual a primeira opção em serviço de saúde quando as crianças adoecem e as respostas concentraram-se entre as UBSs com 42,3\%, seguidas pelo PSP/HU com 36\% A utilização de um serviço de urgência como primeira alternativa para tratamento de enfermidades infantis mostrou-se alta e novamente evidenciou-se a utilização inadequada dos serviços. Schraiber (2002) compartilha com essa idéia e argumenta que as unidades de saúde devem funcionar como porta de entrada da clientela ao sistema de saúde. No entanto, na prática o Hospital Universitário funciona, em grande parte dos casos, como principal acesso ao sistema executando papel de UBSs e ambulatório.

Observou-se que a grande maioria das crianças desse estudo $(80,6 \%)$ são usuárias da rede básica, porém, a procura por programas de prevenção e promoção à saúde geralmente, é baixa conforme se verifica pela tabela 5, a qual evidencia que somente $36,3 \%$ dos entrevistados referiram utilizar as UBSs para cuidados de atenção básica como puericultura, vacinação, enquanto 49,8\% das crianças acessam a unidade básica para consultas com o pediatra em horário marcado. Carvalho (1998) apóia esse achado ao relatar em seu estudo que a procura predominante na rede básica não é por ações de prevenção, que precedem à doença, mas sim ações visando à cura, confirmado pela busca por atendimento médico.

\section{Considerações finais}

Após a análise dos resultados, comprovouse de diferentes maneiras, que a população não usa, predominantemente, a atenção básica como primeira opção de atendimento à saúde de seus filhos indicando descrédito ou subvalorização da mesma, e utiliza serviço de alta complexidade como porta de entrada ao sistema de saúde. Este estudo evidencia, ainda, o predomínio de um modelo curativo, hospitalocêntrico e centrado no médico. Várias questões apontaram a baixa resolutividade e eficácia dos serviços, recomendando expressamente a necessidade de (re) organização dos serviços de saúde que atende a criança no município. Embora se admita que o SUS ainda é uma construção, e esse processo seja historicamente determinado, essa mudança apenas ocorrerá com a transformação de toda conjuntura: dos profissionais, dos serviços e da população.

Com este levantamento de dados acredita-se que os profissionais da área da saúde, em especial os da rede básica devem em conjunto, acolher os usuários, 
de forma a criar vínculo entre a população e equipe de saúde, além de garantir maior resolutividade das ações, gerando maior confiança e quem sabe dessa forma a população utilizaria com maior freqüência a UBS como porta de entrada no sistema de saúde respeitando a hierarquização e complexidade dos diferentes serviços.

Sugere-se ainda que realizem estudos buscando conhecer o ponto de vista de usuários dos outros serviços como PAI e UBSs, além de investigar-se a forma como a população é acolhida e atendida na rede básica.

\section{Referências}

BARACAT, E. C. E. Febre. In: GRISI, S. J. F. E. et al. Estratégia atenção integrada às doenças prevalentes da infância AIDPI. Washington: OPAS, 2005. Cap. 17, p.207-216.

BRASIL. Ministério da Saúde. Agenda de compromissos para saúde integral da criança e redução da mortalidade infantil. Brasília: o Ministério, 2005.

CARVALHO, A. I; GOULART, F. A. A; RIBEIRO, J. M.; MALACHIAS, I. (Org.). Gestão de saúde: curso de aperfeiçoamento para dirigentes municipais de saúde. Rio de Janeiro: FIOCRUZ; Brasília: UNB, 1998.

CECILIO, L. C. O. Modelo tecno-assistenciais em saúde: da pirâmide ao círculo, uma possibilidade a ser explorada. Caderno de Saúde Pública, Rio de Janeiro, v.13, n.3, p.469-478, jul./set. 1997.

CRUZ, D. C. O perfil das crianças atendidas num pronto socorro pediátrico de um hospital universitário pode subsidiar a análise da assistência à saúde infantil? 2005. Trabalho de Conclusão de Curso (Departamento de Enfermagem) - Universidade Estadual de Londrina, Londrina.
FURTADO, B. M. A. S. M.; ARAÚJO, J. L. C.; CAVALCANTI, P. O perfil da emergência do Hospital da Restauração: uma análise dos possíveis impactos após a municipalização dos serviços de saúde. Revista Brasileira de Epidemiologia, São Paulo, v.7, n.3, p. 279-289, set. 2004.

KOVACS, M. H.; FELICIANO, K. V. O.; SARINHO, S. W.; VERAS; A. A. C. A. Acessibilidade às ações básicas entre crianças atendidas em serviços de pronto-socorro. Jornal de Pediatria (Rio de Janeiro), Porto Alegre, v. 81, n. 3, maio/jun. 2005. Disponível em: <http://www. scielo.br/scielo.php?script $=$ sci_arttext\&pid $=$ S0021$75572005000400013 \& \operatorname{lng}=\mathrm{pt} \& \mathrm{nrm}=\mathrm{iso}>$. Acesso em: 8 ago. 2006.

MALTA, D. C.; CECÍlIO, L. C. O; MERHY, E. E.; FRANCO, T. B.; JORGE, A. O.; COSTA, M. A. Perspectivas da regulação na saúde suplementar diante dos modelos assistenciais. Ciência e Saúde Coletiva, Rio de Janeiro, v. 9, n. 2, p. 433-444, abr.jun. 2004.

MARTIN, V. B.; ANGELO, M. A organização familiar para o cuidado dos filhos: percepção das mães em uma comunidade de baixa renda. Revista Latino-Americana de Enfermagem, Ribeirão Preto, v. 7, n. 4, p. 89-95, out. 1999.

ROCHA, S. M. M.; LIMA, R. A. G.; SCOCHI, C. G. S.; VENDRÚSCULO, D. M. S.; MELLO, D. F. Estudo da assistência integral à criança e ao adolescente através da pesquisa qualitativa. Revista. Latino-Americana de Enfermagem, Ribeirão Preto, v. 6, n. 5, p. 5-15, dez. 1998.

SCHRAIBER, L. B. O centro de saúde. In. ISSLER, H.; LEONE, C.; MARCONDES, E. (Coord.).

Pediatria na atenção primária. São Paulo: Sarvier, 2002. Cap. 1, p. 3-13.

TRAVASSOS, C.; MARTINS, M. Uma revisão sobre os conceitos de acesso e utilização de serviços de saúde. Caderno de Saúde Pública, Rio de Janeiro, v. 20 supl. 2, p.190-198, 2004.

VIEIRA, M. A.; LIMA, R. A. G. Crianças e adolescentes com doença crônica: convivendo com mudanças. Revista Latino-Americana de Enfermagem, Ribeirão Preto v. 10, n. 4, p. 552-560, jul./ago. 2002. 
